\title{
Neuronal Induction and Bioenergetics Characterization of Human Forearm Adipose Stem Cells from Parkinson's Disease Patients and Healthy Controls
}

Ingrid González-Casacuberta

Biobanc de I'Hospital Clinic IDIBAPS BT: Biobanc de I'Hospital Clinic IDIBAPS

Dolores Vilas

Hospital Germans Trias i Pujol: Hospital Universitari Germans Trias i Pujol

Claustre Pont-Sunyer

Granollers General Hospital: Hospital General de Granollers

\section{Ester Tobías}

Universitat de Barcelona

Judith Cantó

Hospital Clinic de Barcelona

\section{Laura Valls-Roca}

Universitat de Barcelona

\section{Francesc Josep García-García}

CIBERER: Centro de Investigacion Biomedica en Red de Enfermedades Raras

\section{Glòria Garrabou}

Universitat de Barcelona

Josep Maria Grau-Junyent

Hospital Clinic de Barcelona

\section{Maria Josep Martí}

Hospital Clinic de Barcelona

\section{Francesc Cardellach}

Hospital Clinic de Barcelona

Constanza Morén ( $\square$ cmoren1@clinic.cat)

Hospital Clinic Biobank IDIBAPS: Biobanc de l'Hospital Clinic IDIBAPS https://orcid.org/0000-0001-68487407

\section{Research}

Keywords: Adipose stem cells, bioenergetics, human, neuronal transdifferentiation, Parkinson's disease.

Posted Date: August 3rd, 2021 
DOI: https://doi.org/10.21203/rs.3.rs-754234/v1

License: (c) (1) This work is licensed under a Creative Commons Attribution 4.0 International License. Read Full License

Version of Record: A version of this preprint was published at PLOS ONE on March 15th, 2022. See the published version at https://doi.org/10.1371/journal.pone.0265256. 


\section{Abstract}

Background: Neurodegenerative diseases, such as Parkinson's disease, are heterogeneous disorders with multifactorial nature involving impaired bioenergetics; that are on the rise with the increasing global population and average lifespan. Without definite therapeutic options, both stem regenerative medicine and bioenergetics have been proposed as promising therapeutic targets in the neurologic field. The rationale of the present study was to assess human derived adipose stem cells (hASC) potential to transdifferentiate into neuronal-like cells (NhASC and neurospheres), as well as to explore hASC bioenergetic profile.

Methods: To this purpose, hASC derived from the forearm of both healthy controls and clinical diagnosed Parkinson's disease patients (PD) were included in this study and transdifferentiated through neuronal induction.

Results: Morphological, growth features and neuronal protein expression markers of differentiated neuronlike NhASC and neurospheres were achieved. Increased MAP-2 neuronal marker protein expression upon neuronal induction ( $p<0.05$ from undifferentiated hASC vs. 28 and 36 days of differentiation) and increased blll tubulin neuronal marker protein expression upon neuronal induction $(p<0.05$ from undifferentiated hASC vs. 6,28 and 36 days of differentiation) were found. Bioenergetic profile was detectable through high resolution respirometry approaches in hASC but did not lead to differential oxidative capacity rates in healthy or clinical diagnosed PD hASC.

Conclusions: We have confirmed the capability of transdifferentiation to neuronal-like profile of hASC derived from the forearem of human subjects and characterized the bioenergetic oxidative profile of hASC. Despite the latter did not lead to significant differential respiration profiles, trends to suboptimal maximal respiratory capacity in PD were found. The neuronal induction leading to positive neuronal protein expression markers is a relevant issue that encourages the suitability of the NhASC models in neurodegeneration.

\section{Introduction}

Neurodegenerative diseases, including Parkinson's disease (PD), Alzheimer's disease, or amyotrophic lateral sclerosis (ALS), among many others, are a heterogeneous group of debilitating disorders with multifactorial etiologies and pathogenesis. Their prevalence is on the rise with the increasing global population and average lifespan; and, despite many treatment approaches have been tested, there are currently no effective preventive or clear therapeutic options. Multifactorial nature of such disorders involves different molecular with impaired bioenergetics. Indeed, mitochondrial dysfunction, directly implicated in the cell bioenergetics, is emerging as an important feature in the etiopathogenesis of these age-related neurodegenerative diseases. Previous findings of our group demonstrated suboptimal bioenergetics profile in a fibroblast models derived from in different genetic forms PD patients (1-3).

Adult stem cells are multi-potent undifferentiated cells found in some tissues of the adult organisms. These cells are capable of self-renewal and differentiate into specialized cells and represent a promising tool for replacement cell therapy purposes in a panoply of diseases, including neurodegenerative disorders. Growing 
body of evidence underpinning the potential of stem cell approaches in neurodegeneration is continuously emerging in the recent literature, such as the finding that dental pulp stem cells stimulate neuronal differentiation of PC12 cells (4).

From adult stem cells, adipose stem cells are also considered a highly encouraging implement for regenerative medicine (5). Adipose tissue is the most abundant and accessible source of adult stem cells. The relatively minimal invasive collection procedure, the adult stem cells ability for differentiation into different cell lineages, as well as their safe autologous transplantation are the factors that allow adipose stem cells as an alternative source for bone marrow cells (6). Adipose tissue is composed of mature adipocytes and heterogenous cell population including stromal vascular fraction (7). The vascular fraction is consisted of various cell types, such as immune cells, fibroblasts, pericytes, endothelial cells, and adipogenic progenitor stromal cells, which attach together by collagen fibers (5). In culture, human adiposederived stem cells (hASC) express cell surface markers that are similar to those expressed by mesenchymal stem cells, including CD105, SH3, CD90, and CD44; however, they do not express the hematopoietic marker CD45 and the endothelial marker CD31 $(8,9)$. As adipose tissue contains such a heterogenous population of partially differentiated cells of adipocyte lineage, tissue repair, angiogenesis and neovascularization may be closely linked to the function of hASC in a complex relationship (10). Due to such convoluted interactions and heterogenous features within the adipose tissue, specific biological mechanisms involved in hASC proper functioning and homeostasis, including stem pluripotency/transdifferentiation capacities as well as bioenergetic fingerprint, remains unknown. Interestingly both stem (11) and bioenergetic (12) tools, explored in the present study, have been proposed as promising therapeutic novel targets in the neurologic field.

Epigenesis has been reported to be involved in the cellular differentiation during development, which is controlled by different factors such as growth and environmental factors (13). These factors are involved directly or indirectly in the transcription and expression of genes by genetic reprogramming process (14). For example, adipose derived stem cells could be induced into neural and glial phenotypes by transdifferentiation mechanism (11).

There are convincing findings demonstrating the relevance of nervous regulation of adipose tissue on metabolic and secretory activities, including plasticity (proliferation, differentiation, trans-differentiation, apoptosis) $(15,16)$. The neuronal feedback cycle between adipose tissues and brain plays a crucial role in energetic homeostasis. The oxidative and lipolytic metabolic nature of neuronal and adipose tissues, respectively, confers a higher level of complexity in their relationship and in the molecular studies carried out in both biologic sources and derived cell models, especially, those related to bioenergetics. Although little is known about bioenergetic profile of hASC, a recent study exploring hASC bioenergetics demonstrated for the first time, that this stem cells can affect metabolic homeostasis by promoting damaged mitochondrial clearance through mitophagy, thereby delaying aging (17).

The rationale of the present study is to assess hASC potential to transdifferentiate into neuronal-like cells (NhASC and neurospheres), together with the assessment of their bioenergetic fingerprint. To this purpose, 
hASC derived from both healthy controls and clinical diagnosed PD $(P D)$ patients were included in this study.

The present work contributes to knowledge of characterization of a stem cell model and their bioenergetic profile as potential useful novel target for treating the neurodegenerative condition.

\section{Methods}

\section{Subjects and Sample Collection}

Samples from healthy controls $(n=3)$ and from patients with PD $(n=3)$ were used in this study; written informed consent was obtained before subcutaneous fat was collected. The protocols used for collection were approved by the Hospital Clínic Ethics Committee (HCB EC number 2018/0228). Skin-punch biopsies containing subcutaneous adipose tissue ( $4 \mathrm{~mm}$ in diameter) were obtained from the forearm of participants; the subcutaneous adipose tissues were dissected from skin and used for cell isolation. Human subcutaneous adipose tissues were washed once with growth medium and dissected into small pieces (less than $0.2 \mathrm{~cm}$ diameter). E. g. from a given biopsy, 18 pieces could be obtained.

\section{Adipose cell culture and mainteinance}

The small pieces of adipose tissues were explanted in sterile $10 \mathrm{cc}$ dishes. A sterilized coverslip was used to cover the tissue in order to prevent tissue floating in the medium. Complete media for hASC consisted of Dulbecco's Modified Eagle Medium (DMEM) supplemented with 10\% foetal bovine serum (FBS), 1\% nonessential amino acids (NEAA), $1 \%$ pyruvate and $0.5 \%$ penicillin-streptomycin antibiotics. Media were changed every 3 days until migrated cells reached $70-80 \%$ confluency. Then, the cells were dissociated from the well with accutase (Millipore) and seeded in a ratio of 1:3 in normal culture dishes with growth medium. The passage was conducted as follows: medium was removed; cells were washed with sterile $1 \times$ PBS and were incubated with accutase $(1 \mathrm{~mL} /$ dish; $100 \times 20 \mathrm{~mm})$ at $37^{\circ} \mathrm{C}$ for $3-5 \mathrm{~min}$. Growth medium was added to the dissociated cells and the medium containing cells was divided over three individual dishes. Cells were cultured and maintained in growth medium at $37^{\circ} \mathrm{C}$ with $5 \% \mathrm{CO}_{2} 95 \%$ air.

\section{Neurosphere hASC transdifferentiation}

When to $70-80 \%$ confluency was reached, hASC were collected and pelleted by centrifugation at $2000 \mathrm{~g}$ for $10 \mathrm{~min}$. The cell pellet was resuspended with neurosphere media, transferred to an uncoated 12-well plate for further maintenance and experiments, and incubated at $37^{\circ} \mathrm{C}$ with $5 \% \mathrm{CO}_{2} 95 \%$ air. Cells were cultured in DMEM-F12 (1:1) containing the supplements and growth factors: 1x B27 (Thermo Fisher catalog $\mathrm{n}^{\circ}$ \#17504-044), 20 $\mu \mathrm{L}$ leukemia inhibitor (10ng/mL), basic fibroblast growth factor (40ng/mL FGF2 or bFGF, catalog $\mathrm{n}^{\circ} \# 233$-FB-025), epidermal growth factor (10ng/mL EGF) and 1\% penicillin-streptomycin antibiotics were harvested in sterile 6-well plates. Neurospheres formed within $24 \mathrm{~h}$ after induction with the neurosphere media. The neurospheres were cultured and maintained in the same conditions changing media every 5 days.

\section{Neuron-like hASC transdifferentiation (NhASC)}


On day 1, adipose stem cells were seeded at 8000 cells $/ \mathrm{ml}$ density and $3 \mathrm{ml}$ were harvested on a 6-well plate previously coated with fibronectin (Sigma \#F1141), 1 $\mathrm{mg} / \mathrm{ml}$. On day 2, h-ASC media was removed and substituted by differentiation neurobasal medium (Thermo Fisher catalog $n^{\circ} \# 21103-049$ ) containing B-27 (Thermo Fisher catalog $\left.n^{\circ} \# 17504-044\right), 250 n g / m l$ human sonic Hedgehog (sHH, catalog $n^{\circ} \# 1845-$ $\mathrm{SH}-025), 100 \mathrm{ng} / \mathrm{ml}$ human fibroblast growth factor 8 (FGF8, catalog $\left.\mathrm{n}^{\circ} \# 423-\mathrm{F} 8-025\right), 50 \mathrm{ng} / \mathrm{ml}$ human basic fibroblast growth factor (bFGF, catalog $\left.n^{\circ} \# 233-F B-025\right)$ and antibiotic/antimycotic solution (Sigma catalog $\mathrm{n}^{\circ}$ \#A5955). On day 12, $1 \mathrm{ml}$ of medium was apirated and $2 \mathrm{ml}$ of differentiation medium containing 50ng/ml human BDNF (catalog $\left.\mathrm{n}^{\circ} \# 248-B D-025\right)$ was added. This last step was repeated every 8 days (on days 20, 28, 36 and 40 ) and NhASC cells were kept until maximum 42-46 days.

\section{Flow Cytometry}

Cells were harvested, washed, and centrifuged for $5 \mathrm{~min}$ at $300 \mathrm{~g}$. Pellets were resuspended in sterile complete media and cell counts were determined. Approximately 500.000 cells were used for each reaction, including different cell types for comparison purposes (fibroblasts and hASC). Cells were recorded on the BD LSR II flow cytometer (BD Biosciences, Oxford, UK) using BD FACSDiva software, and data were analyzed using FlowJo software (TreeStar., Ashland, OR, USA) (18). The following antibody panel was used for hASC characterization: APC mouse anti-human CD45 (BD Biosciences catalog $n^{\circ} \# 555485$ ), PE mouse anti-human CD34 (BD Horizon, BD Biosciences, catalog $n^{\circ}$ \#562577), PE mouse anti-human CD73 (BD Biosciences catalog $\left.n^{\circ} \# 550257\right), P E$ mouse anti-human CD90 (Biosciences catalog $n^{\circ}$ \#555596) and PE mouse anti-human CD-105 (BD Biosciences catalog $n^{\circ} \# 560839$ ). The percentage of fluorescence in hASC was determined in all cases.

\section{Western blot analysis and densitometry}

Western blot experiments were conducted as reported elsewhere (19). Briefly, hASC and NhASC were lysed and placed on ice for 15 min with RIPA buffer supplemented with 1x Halt protease inhibitor cocktail (Pierce). Cell lysates were centrifuged at $21,000 \mathrm{~g}$ at $4^{\circ} \mathrm{C}$ for $5 \mathrm{~min}$ and soluble material was retained for western blot analysis. Protein levels were determined by using a bicinchoninic acid (BCA) kit (Pierce Thermo Fisher; Basingstoke, UK) as described (20). Equal amounts of 20-40 $\mu$ g protein from the soluble material were resolved under reducing conditions in either: i) NuPAGE 4-12\% polyacrylamide precast gels (Invitrogen, Carlsbad, CA, USA) using the 2 (N morpholino) ethane sulphonic acid (MES) buffer or: ii) NuPAGE 12\% (Invitrogen, Carlsbad, CA, USA) using MOPS buffer and transferred onto Immobilon polyvinylidene difluoride membrane (PVDF) or nitrocellulose (Millipore, Watford, UK). Blocking was made with $10 \%$ skimmed milk powder in PBS. Antibodies detecting microtubule associated protein 2 (MAP2) (1:1000 MAP2, Invitrogen), $\beta$ III tubulin (1:1000 ab7751 Abcam), $\beta$ actin (1:10000, Sigma) were used. Horseradish peroxidase conjugated secondary antibodies against mouse and rabbit IgG were used (1:2000) (Dako, Glostrup, Denmark). ECL reagent (GE Healthcare, Bucks, UK) was used to develop the blots and signals detected through LAS4000 system and band densities measured using ImageJ and ImageQuant analysis software.

\section{High resolution respirometry studies}

High resolution assessment of basal respiration, proton leak, maximal respiration and residual respiration was conducted through Oroboros and Seahorse technologies. 


\section{Oxidative assessment (Oroboros 2k-oxygraph)}

The Oxygraph-2k (02k, OroborosTM instruments, Innsbruck, Austria) for high-resolution respirometry is a closed two-chamber respirometer for high-resolution respirometry $(21,22)$. The $02 k$ is a barometric pressure transducer enables automatic oxygen calibrations implemented in the DatLab software. Oxygen consumption by hASC samples suspended in $2.1 \mathrm{~mL}$ of MIR05 solution was monitored under controlled temperature $\left(37^{\circ} \mathrm{C}\right)$ and stirring $(300 \mathrm{rpm})$. The main respiratory states were determined by routine, the natural basal respiration of intact cells in the absence of substrate/inhibitor addition; then the proton leak (no-phosphorylation), after ATP synthase inhibition by oligomycin $(1 \mu \mathrm{g} / \mathrm{mL})$, which blocks ATP synthase allowing the assessment of the natural proton leak across the inner mitochondrial membrane (IMM). Maximal respiratory capacity, so-called ETS, in the presence of the mitochondrial uncoupler carbonyl cyanide m-chlorophenylhydrazone (CCCP, 1 or $2 \mu \mathrm{M})$. CCCP is an ionophore that directly transports protons across the IMM bypassing the ATP synthase proton channel thus leading to a rapid consumption of oxygen without the ATP generation. Finally, residual respiration, so-called Rox, after complex III inhibition by antimycin $\mathrm{A}(\mathrm{AA}, 3 \mu \mathrm{M})(23)$. After the oxygen consumption measurements, the reaction mixture was completely removed from the oxygraph chamber and submitted to protein BCA method (20) leading to $\mathrm{pmol} / \mathrm{min}$.ug protein units.

\section{Oxidative assessment (Agilent Seahorse instrument XFe24 Analyzer)}

Oxygen consumption rates (OCRs) were measured in intact adherent hASC through Agilent SeahorseTM XFe24 Analyzer (Seahorse Bioscience, Wilmington, DE, USA), using the Cell Mito Stress Test (24). Briefly, 15,000-30,000 hASC/per well were seeded in customized 24-well Seahorse cell culture plates and left to adhere overnight in $250 \mathrm{uL}$ of growth medium. Each cell line was seeded in triplicate per condition. After 24 hours, growth medium was removed and wells were washed and replaced with Seahorse XF Base Medium (Seahorse Bioscience). Afterwards, plates were incubated for $30 \mathrm{~min}$ at $37^{\circ} \mathrm{C}$ without $\mathrm{CO}_{2}$ according to manufacturer's protocol. In order to determine the different respiratory control ratios, oxygen consumption was measured under basal conditions and after the addition of oligomycin, to assess proton leak. Similary to previously described CCCP tritiation in the Oroboros technology, this was followed by the addition of the uncoupler carbonyl cyanide-4-(trifluoromethoxy) phenylhydrazone (FCCP) to measure the maximal respiratory capacity. FCCP is an ionophore that directly transports protons across the IMM bypassing the ATP synthase proton channel thus leading to a rapid consumption of oxygen without the ATP generation. Finally, complex I and III inhibitors, rotenone and antimycin-A, were added to assess unspecific nonmitochondrial respiration (all reagents from Sigma-Aldrich). In addition, spare respiratory capacity and basal/maximal respiratory ratio were calculated as the maximal after basal OCR subtraction expressed in percentage, and the ratio between basal and maximal OCR, respectively. All respiration values were normalized to total cell protein content determined in each well through the BCA assay (20) leading to $\mathrm{pmol} / \mathrm{min}$.ug protein units.

\section{Results}




\section{Characterization of hASC and ability to differentiatiation in neurobasal media}

Adipose tissue explants led to proliferating hASC after roughly 3 weeks of biopsy processing (Fig. 1A). For unknown reasons some samples did not lead to successful proliferative hASC. Mesenchymal stem cluster differentiation CD73, CD90 and CD105 markers showed positivity in hASC through flow cytometry assessment. All proliferating cell lines, including controls and cases, developed similarly (Fig. 1B, upper and lower panels, respectively) leading to elongation and branching shapes during the differentiation stages (from left to right in all cases). hASC also enabled to neurosphere formation after 4 days of induction with neurosphere media (Fig. 1C, from left to right). As expected, blll tubulin neuronal marker staining in undifferentiated hASC did not lead to detectable signal (Fig. 1D, first panel), whereas the signal was present in hASC exposed to neurobasal differentiation media after 20 days of differentiation (Fig. 1D, middle panel). Other neuronal markers were also tested, such as tyrosine hydroxilase, microtubule-associated-protein-2 (MAP-2) and dopamine transporter, leading to unconclusive results, due to high background unspecific fluorescence detection. Neurosphere immunostaining options were limited due to the tridimensional structure of the aggregates, although cytoplasmatic cell and mitochondrial structural networks could be determined by $b$ actin and TOM20, respectively (Fig. 1D, third panel).

\section{hASC and NhASC proliferation capacity}

NhASC showed lower proliferative capacity than undifferentiated hASC, suggesting postmitotic neuronallike features with less growth capacity (Fig. 2B). All proliferating controls and cases presented the same growth capacities in both basal and differentiating conditions.

\section{Neuronal differentiation protein markers}

Immunodetection towards different neuronal markers was conducted. Dopaminergic markers tyrosine hydroxilase and dopamine transporter DAT did not lead to conclusive results due to absence of signal in all the samples, whereas microtubule associated protein MAP-2 marker of mature non-proliferative neuronal cell led to positive staining, specifically in the last period of differentiation, similarly to blll tubulin, an early biomarker of neural cell differentiation from multipotent progenitors (25), which led to significant increase from 6 day of differentiation.

\section{Oxidative capacity of hASC control and pathological lines}

\section{High resolution respirometry through Oroboros and SeahorseX24}

Mitochondrial energy metabolism can be tested through Oroboros technology comprehensively in real time by titrating various substrates, uncouplers, inhibitors, and other substances during the experiment. First, oxidative profile of control hASC was assessed compared to pathological PD hASC. Basal respirometry, proton leak after oligomycin addition, maximal respiratory capacity through uncoupler titration and residual respiratory activity after inhibition of mitochondrial respiratory chain were determined (Fig. 4AB). Non- 
significant trends towards lower basal respiratory performance in intact cells were observed in the PD hASC compared to controls. In order to further confirm such observed trends in basal respiration of PD cases, further oxidative experimental assessments using 24-well Seahorse Agilent technology were conducted. Similarly to previous findings, Seahorse data including basal, leak, maximal and residual respiratory capacity, led to comparable results confirming the lack of differences between controls and PD hASC, as well as the suitability of hASC to undergo high resolution respirometry analysis (Fig. 4CD).

Despite lack of significant differences in respirometry measurements, trends towards suboptimal maximal respiration capacity in PD hASC were confirmed in both oxygraphs compared with the healthy control hASC lines.

Further respirometry parameters were also explored in order to confirm oxidative state of control and PD hASC leading to non-significant differences between both groups (Fig. 5). Due to the consistent lack of differences from all approaches and oxidative parameters in basal undifferentiated hASC vs. neuronal transdifferentiated NhASC, oxidative analysis were not conducted in differentiated control vs. PD NhASC lines.

The dataset supporting the conclusions of this article are included within the article and its additional file 1.

\section{Discussion}

The present study reports the characterization of hASC considering their ability to neuronal-like transdifferentiation and their bioenergetics fingerprint. Two neuronal-like transdifferentiation procedures were adequately performed by cultured hASC derived from the forearm human healthy controls and PD cases. First, neuronal-like features were found in cultured NhASC under exposure to neurobasal differentiation media. Morphologically, branching and elongation of NhASC were observed upon chemical induction, as already depicted in the literature (26). On the other hand, expression of early and mature neuronal protein markers, blll tubulin and MAP-2 respectively, were observed in the NhASC model and were stable during several days of differentiation. The increase of blll expression was achieved at 6 days of neuronal differentiation whereas the increase of MAP-2 was reached at 28 days of neuronal differentiation. The time period needed to detect increase of such protein expression markers varied as expected, taking into account that blll tubulin is an indicator of early neuronal models (25) and MAP-2 is a biomarker for mature neuronal models (27). Second, adequate neurosphere generation was observed under exposure to neurosphere induction media, as previously reported $(19,28)$. Neurosphere generation was conducted as an additional test of another neuronal transdifferentiation potential capacity of the hASC model in this study, leading to proper neurosphere generation. In addition, the characterization of the hASC included the assessment of the oxidative profile in order to decipher bioenergetics fingerprint of the model, leading to similar trends between healthy controls and PD cases. So far, this is in contrast with suboptimal bioenergetics reported in other PD models (1), although comparable basal respiration has also been described in PD cybrid models compared to controls (29) and, similarly to this study, non-significant trends have also been described in hASC in the literature (17). Despite lack of significant differences in 
respirometry measurements, trends towards suboptimal maximal respiration capacity in PD hASC were confirmed in both oxygraphs compared with the healthy control hASC lines.

Limited sample inclusion is probably most likely the reason for comparable preserved results in bioenergetic assessment of the hASC model, but this not precluded of the relevant finding related to positive neuronal protein expression upon induction of the model.

To gain knowledge in biological mechanisms of hASC is an urgent need, as they represent promising therapeutic tools for a variety of disorders. Recently hASC have been described to protect motor neurons and reduce glial activation in both in vitro and in vivo models of ALS (30). Effectiveness and mechanisms of adipose-derived stem cell therapy in animal models of PD have been already depicted in the literature. Transplantation of ASC is a therapeutic option that shows long-lasting effects in animal models of PD; following the transplantation of ASC, tyrosine hydroxylase-positive neurons have been described to recover in the lesion (31). The potential mechanisms of ASC involve neurogenesis and neuroprotective effects and the standardized induction of neural form of transplanted ASC can lead to a future application in clinical practice. Unfortunately, in our hASC transdifferentiation model we could not either detect tyrosine hydroxylase positive or any other dopamine related markers, such as DAT. In this context, standardization of optimal transdifferentiation procedures is an urgent need. Optimal conditions for hASC procedures are still a matter of debate in a field that is gaining strength as well as remains to be explored. For instance, in a recent study, hASC under hypoxic culture conditions was described to improve neuronal differentiation and nerve repair (32). Transdifferentiation ability of human bone marrow derived stem cells to neurons upon chemical induction had been previously described by Woodbury's et al $(33,34)$. As mesenchymal stem cells share many biological features with ASC, this protocol has been adapted for the neural ASC differentiation with different modifications. This well-known method has been experimentally revisited several times as the stage-specific incidence of ASC differentiation needs to be validated (26). Although optimal procedure for hASC transdifferentiation to neural lineages need to be better defined, which may represent a limitation for this and other similar studies, the protocol for neuronal ASC induction herein has been previously reported (35). Further refinement of the protocols and study of the occurrence and terminal differentiation of neuroprogenitors would identify a promising source for neural tissue replacement and is warranted. Yet, the main limitation of this study was the limited sample size regarding both source of sample, which probably hampered for successful proliferative cultures (the low amount of tissue led to not proliferative cell cultures in some cases) and regarding of the number of subjects included in the study, which may mask differential bioenergetics profile between controls and pathological lines (the low number of subjects was likely to promote loss of statistical power). Indeed the forearm origin of our samples led to limited source of biological material, but suitability of the use of adipose tissue should also be considered as an advantage for the present model. In fact, amongst the various types of mesenchymal stem cells, hASC are relatively easy to obtain through subcutaneous fat aspiration, liposuction, other surgery interventions and methods (5). Compared with harvesting bone marrow stem cells, this process is less invasive and less controversial than the use of embryonic stem cells. In addition, transdifferentiation allows direct differentiation properties as an advantage that is absent in other cell models such as induced pluripotent stem cells (IPSC) (36), although potential neurodevelopmental features may not be detected in this model compared with IPSC. 
In summary, we have confirmed the capability of transdifferentiation to neuronal-like profile of hASC derived from the forearem of human subjects and characterized the bioenergetic oxidative profile of hASC which did not lead to differential respiration performances in healthy controls and PD patients. Limited sample size could explain the lack of statistical significance, and further studies following that direction are needed.

\section{Conclusions}

Human adipose stem cells derived from the forearm of the subjects of study allow for neuronal transdifferentiation and bioenergetics assessment and may therefore represent a suitable tool to model and investigate molecular processes related to Parkinson's disease and other neurodegenerative processes as well as in order to test potential pharmacological strategies.

\section{Abbreviations}

ALS- Amyotrophic lateral sclerosis

AU- Arbitrary units

CCCP- lonophore carbonyl cyanide m-chlorophenyl hydrazone

CD- Cluster of differentiation

CDPD- Clinical diagnosed Parkinson's disease

DAT- Dopamine transporter

DMEM- Dulbecco's Modified Eagle Medium

Dod-Days of development

FCCP- Ionophore carbonyl cyanide-p-trifluoromethoxyphenylhydrazone

hASC- Human derived adipose stem cells

MAP-2- Microtubule-associated-protein-2

Max-Maximal respiration

NEAA- Non essential amino acids

NhASC- Neuronal induced human adipose stem cells

PBS- Phosphate buffer saline

Res- Residual respiration 


\section{Declarations}

\section{ETHICS APPROVAL AND CONSENT TO PARTICIPATE}

Written informed consent was obtained before subcutaneous fat was collected. The protocols used for collection were approved by the Hospital Clínic Ethics Committee (HCB EC number 2018/0228).

\section{CONSENT FOR PUBLICATION}

Not applicable.

\section{AVAILABILITY OF DATA AND MATERIALS}

The data generated or analysed during this study are included in this published article and its supplementary information files.

\section{COMPETING INTERESTS}

The authors report no conflicts of interest.

\section{FUNDING}

Project number: CP042187 (Fundació Privada Cellex). Morén C was supported by a CIBERER postdoctoral contract (Instituto de Salud Carlos III).

\section{AUTHOR'S CONTRIBUTIONS}

IGC and CM conducted experimental tasks with the help of ET. DV, CP selected the clinical and control cases. JM offered clinical supervision. LVR, JC, FGG and GG reviewed the work and helped with experimental procedures. MJM supervised clinical subjects and the design of the study. CM and FC are the principal investigators of the study. All the co-authors participated in the review process of the manuscript.

\section{ACKNOWLEDGMENTS}

We are grateful to Fundació Privada Cellex for finantial support through the project CP042187 (Fundació Clínic per la Recerca Biomèdica, FCRB, Principal Investigators: Francesc Cardellach and Constanza Morén). Human resources financial support: Ministerio de Ciencia e Innovación and CIBERER, Morén C was supported by a CIBERER postdoctoral contract. We are in debt to Instituto de Salud Carlos III through the 
project [PI17/00359] (Co-funded by European Regional Development Fund/European Social Fund "A way to make Europe".

\section{References}

1. Juárez-Flores DL, González-Casacuberta I, Ezquerra M, Bañó M, Carmona-Pontaque F, Catalán-García $M$, et al. Exhaustion of mitochondrial and autophagic reserve may contribute to the development of LRRK2 G2019S -Parkinson's disease. J Transl Med. 2018;

2. González-Casacuberta I, Juárez-Flores DL, Ezquerra M, Fucho R, Catalán-García M, Guitart-Mampel M, et al. Mitochondrial and autophagic alterations in skin fibroblasts from Parkinson disease patients with Parkin mutations. Aging (Albany NY). 2019;

3. González-Casacuberta I, Morén C, Juárez-Flores DL, Esteve-Codina A, Sierra C, Catalán-García M, et al. Transcriptional alterations in skin fibroblasts from Parkinson's disease patients with parkin mutations. Neurobiol Aging. 2018;

4. Sultan N, Amin LE, Zaher AR, Grawish ME, Scheven BA. Dental pulp stem cells stimulate neuronal differentiation of PC12 cells. Neural Regen Res. 2021;

5. Gimble JM, Katz AJ, Bunnell BA. Adipose-derived stem cells for regenerative medicine. Circulation Research. 2007.

6. Pachón-Peña G, Yu G, Tucker A, Wu X, Vendrell J, Bunnell BA, et al. Stromal stem cells from adipose tissue and bone marrow of age-matched female donors display distinct immunophenotypic profiles. $J$ Cell Physiol. 2011;

7. Li Al, Hokugo A, Jarrahy R, Zuk PA. Human adipose tissue as a source of multipotent stem cells. In: Stem Cells in Aesthetic Procedures: Art, Science, and Clinical Techniques. 2014.

8. Huang T, He D, Kleiner G, Kuluz J. Neuron-like differentiation of adipose-derived stem cells from infant piglets in vitro. J Spinal Cord Med. 2007;

9. Zhang DZ, Gai LY, Liu HW, Jin QH, Huang JH, Zhu XY. Transplantation of autologous adipose-derived stem cells ameliorates cardiac function in rabbits with myocardial infarction. Chin Med J (Engl). 2007;

10. Hutchings G, Janowicz K, Moncrieff L, Dompe C, Strauss E, Kocherova I, et al. The proliferation and differentiation of adipose-derived stem cells in neovascularization and angiogenesis. Int J Mol Sci. 2020;

11. Safford K, Rice H. Stem Cell Therapy for Neurologic Disorders: Therapeutic Potential of AdiposeDerived Stem Cells. Curr Drug Targets. 2005;

12. Onyango IG, Bennett JP, Stokin GB. Regulation of neuronal bioenergetics as a therapeutic strategy in neurodegenerative diseases. Neural Regeneration Research. 2021.

13. Barzilay R, Melamed E, Offen D. Introducing transcription factors to multipotent mesenchymal stem cells: Making transdifferentiation possible. Stem Cells. 2009.

14. Sanchez-Ramos JR. Neural cells derived from adult bone marrow and umbilical cord blood. Journal of Neuroscience Research. 2002. 
15. Bowers RR, Festuccia WTL, Song CK, Shi H, Migliorini RH, Bartness TJ. Sympathetic innervation of white adipose tissue and its regulation of fat cell number. Am J Physiol - Regul Integr Comp Physiol. 2004;

16. Giralt M, Cereijo R, Villarroya F. Adipokines and the endocrine role of adipose tissues. Handb Exp Pharmacol. 2015;

17. Zhang M, Simeng B, Sunrun J, Cao Y, Dong Cao L, Shu G. Adipose-derived stem cells regulate metabolic homeostasis and delay aging by promoting mitophagy. FASEB J [Internet]. 2021;35(7):1-13. Available from: https://faseb.onlinelibrary.wiley.com/doi/10.1096/fj.202100332R

18. Castella M, Boronat A, Martín-lbáñez R, Rodríguez V, Suñé G, Caballero M, et al. Development of a Novel Anti-CD19 Chimeric Antigen Receptor: A Paradigm for an Affordable CAR T Cell Production at Academic Institutions. Mol Ther - Methods Clin Dev. 2019;

19. Morén C, Juárez-Flores DL, Chau KY, Gegg M, Garrabou G, González-Casacuberta I, et al. GBA mutation promotes early mitochondrial dysfunction in 3D neurosphere models. Aging (Albany NY). 2019;

20. Bainor A, Chang L, McQuade TJ, Webb B, Gestwicki JE. Bicinchoninic acid (BCA) assay in low volume. Anal Biochem. 2011;

21. Gnaiger E. Bioenergetics at low oxygen: Dependence of respiration and phosphorylation on oxygen and adenosine diphosphate supply. In: Respiration Physiology. 2001.

22. Pesta D, Gnaiger E. High-resolution respirometry: OXPHOS protocols for human cells and permeabilized fibers from small biopsies of human muscle. Methods Mol Biol. 2012;

23. Long Q, Huang L, Huang K, Yang Q. Assessing mitochondrial bioenergetics in isolated mitochondria from mouse heart tissues using oroboros 2k-oxygraph. In: Methods in Molecular Biology. 2019.

24. Agilent Technologies. Agilent Seahorse XF Cell Mito Stress Test Kit User Guide - Kit 103015-100. https://www.agilent.com/cs/library/usermanuals/public/XF_Cell_Mito_Stress_Test_Kit_User_Guide.pdf. 2019.

25. Sieber-Blu M, Schnell L, Grim M, Hu Y, Schneider R, Schwab M. Characterization of epidermal neural crest stem cell (EPI-NCSC) grafts in the lesioned spinal cord. Mol Cell Neurosci. 2006;32(1):67-81.

26. Radhakrishnan S, Trentz OA, Reddy MS, Rela M, Kandasamy M, Sellathamby S. In vitro transdifferentiation of human adipose tissue-derived stem cells to neural lineage cells - a stage-specific incidence. Adipocyte. 2019;

27. Soltani, MH (Soltani, MH); Pichardo, R (Pichardo, R); Song, ZQ (Song, ZQ); Sangha, N (Sangha, N); Camacho, F (Camacho, F); Satyamoorthy, K (Satyamoorthy, K); Sangueza, OP (Sangueza, OP); Setaluri, V (Setaluri V. Microtubule-associated protein 2, a marker of neuronal differentiation, induces mitotic defects, inhibits growth of melanoma cells, and predicts metastatic potential of cutaneous melanoma. Am J Pathol. 2005;166(6):1841-50.

28. Peng C, Lu L, Li Y, Hu J. Neurospheres Induced from Human Adipose-Derived Stem Cells as a New Source of Neural Progenitor Cells. Cell Transplant. 2019;

29. Esteves AR, Lu J, Rodova M, Onyango I, Lezi E, Dubinsky R, et al. Mitochondrial respiration and respiration-associated proteins in cell lines created through Parkinson's subject mitochondrial transfer. J Neurochem. 2010; 
30. Ciervo Y, Gatto N, Allen C, Grierson A, Ferraiuolo L, Mead RJ, et al. Adipose-derived stem cells protect motor neurons and reduce glial activation in both in vitro and in vivo models of ALS. Mol Ther Methods Clin Dev. 2021;

31. Li K, Li X, Shi G, Lei X, Huang Y, Bai L, et al. Effectiveness and mechanisms of adipose-derived stem cell therapy in animal models of Parkinson's disease: a systematic review and meta-analysis. Translational Neurodegeneration. 2021.

32. Wu S-H, Liao Y-T, Hsueh K-K, Huang H-K, Chen T-M, Chiang E-R, et al. Adipose-Derived Mesenchymal Stem Cells From a Hypoxic Culture Improve Neuronal Differentiation and Nerve Repair. Front Cell Dev Biol. 2021;

33. Woodbury D, Schwarz EJ, Prockop DJ, Black IB. Adult rat and human bone marrow stromal cells differentiate into neurons. J Neurosci Res. 2000;

34. Woodbury D, Reynolds K, Black IB. Adult bone marrow stromal stem cells express germline, ectodermal, endodermal, and mesodermal genes prior to neurogenesis. J Neurosci Res. 2002;

35. Yang SY, Beavan M, Chau KY, Taanman JW, Schapira AHV. A Human Neural Crest Stem Cell-Derived Dopaminergic Neuronal Model Recapitulates Biochemical Abnormalities in GBA1 Mutation Carriers. Stem Cell Reports. 2017;

36. Alzheimer's in a dish - induced pluripotent stem c...: Consulta'l a: [Internet]. [cited 2021 Jul 14]. Available from: http://resolver.ebscohost.com.sire.ub.edu/

\section{Figures}


$1 \mathrm{~A}$
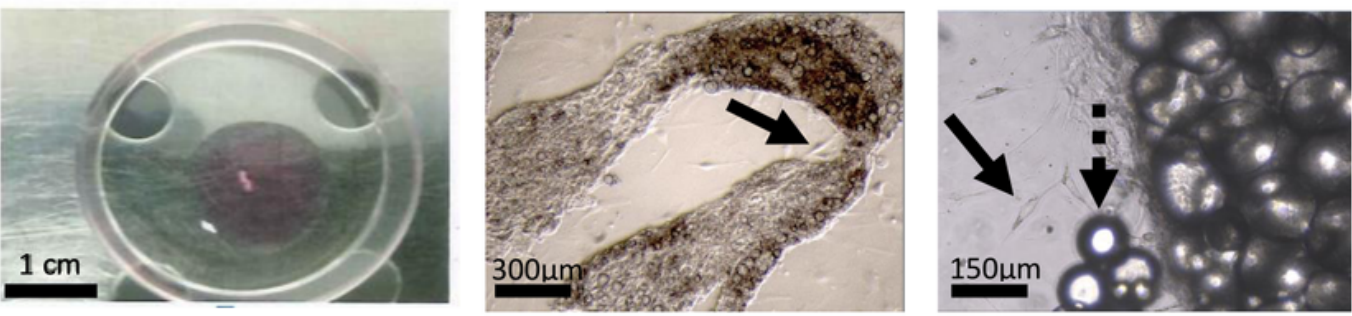

1B
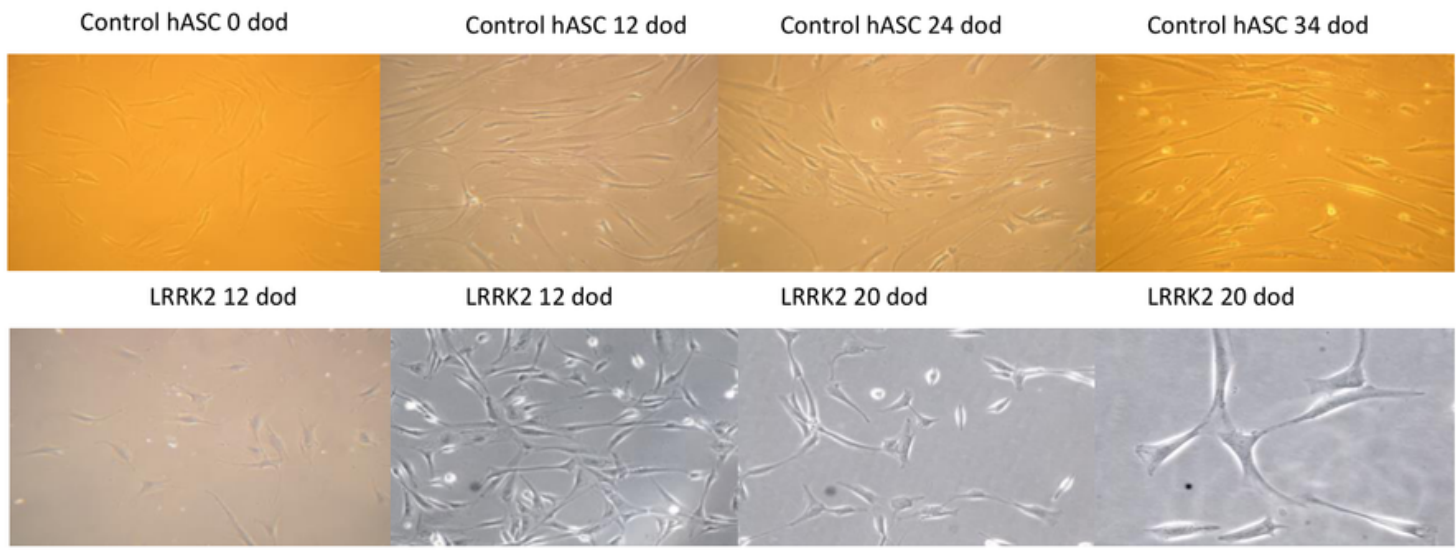

hASC

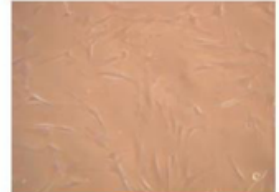

$24 \mathrm{~h}$

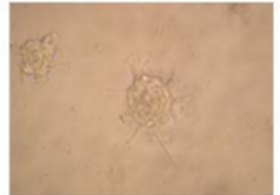

1D

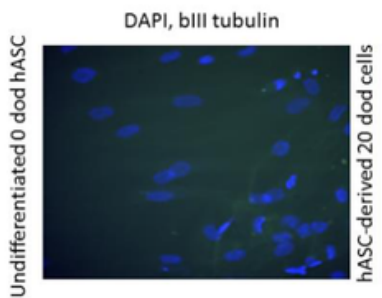

DAPI, b actin, bIII tubulin

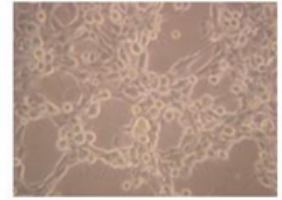

$72 \mathrm{~h}$
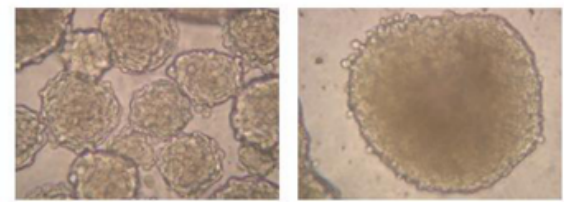

$6 \mathrm{~h}$

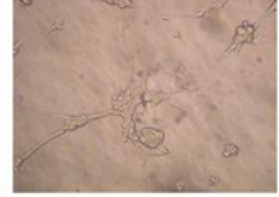

$96 \mathrm{~h}$

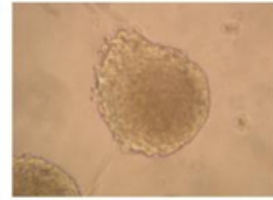

DAPI, b actin, TOM2O
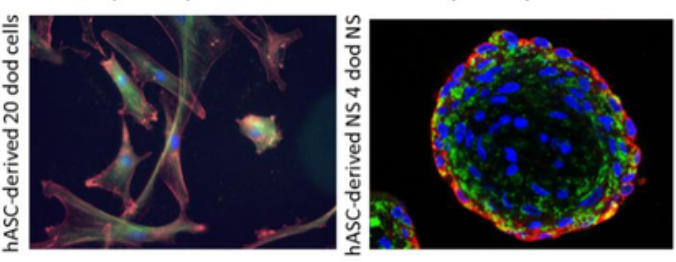

\section{Figure 1}

Representative images of hASC and NhASC. 1A. From left to right, first panel, forearm adipose tissue biopsy on a Petri dish prior to tissue disgregation. Second and third panels, adipose tissue dissected fragments by optical microscopy (5x and 10x respectively). Adipose tissue and scarce adipose cells generated from tissue explant. Black continuous arrow pointing adipose cells coming out from the explant. Black discontinous arrow marking tipical oil drops from adipose tissue samples. 1B. Forearm human adipose stem cells from control and PD-diagnosed lines (upper and lower panels, respectively) over time up to 20 
days of differentiation. 1C. Neurosphere formation over time (hours) and morphology derived from control line of human adipose neural crest stem cells over time up to 4 days of development. Dod, days of development (differentiation), hASC, human adipose stem cells. 1D. Immunofluorescence representative images of forearm human adipose stem cells undifferentiated at basal timepoint conditions (left panel, blue DAPI), after 20 days of differentiation (middle panel, blue DAPI, red $b$ actin, green neuronal blll tubulin) and after neurosphere formation (right panel, blue DAPI, red b actin, green TOM20). hASC, human adipose stem cells.
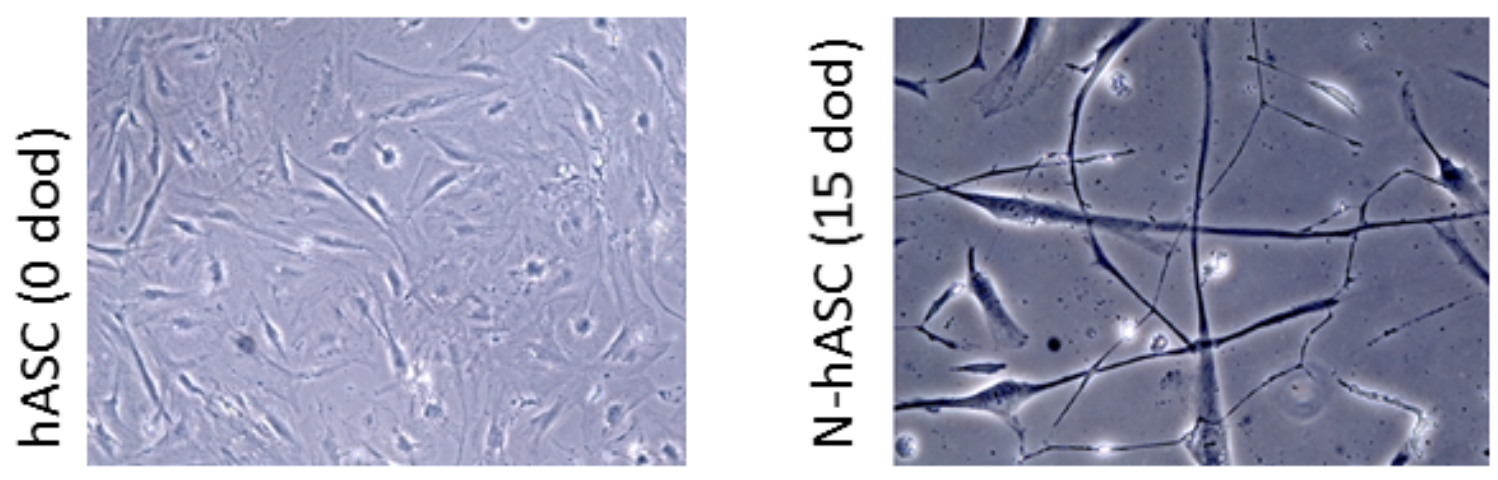

\section{Growth curve}

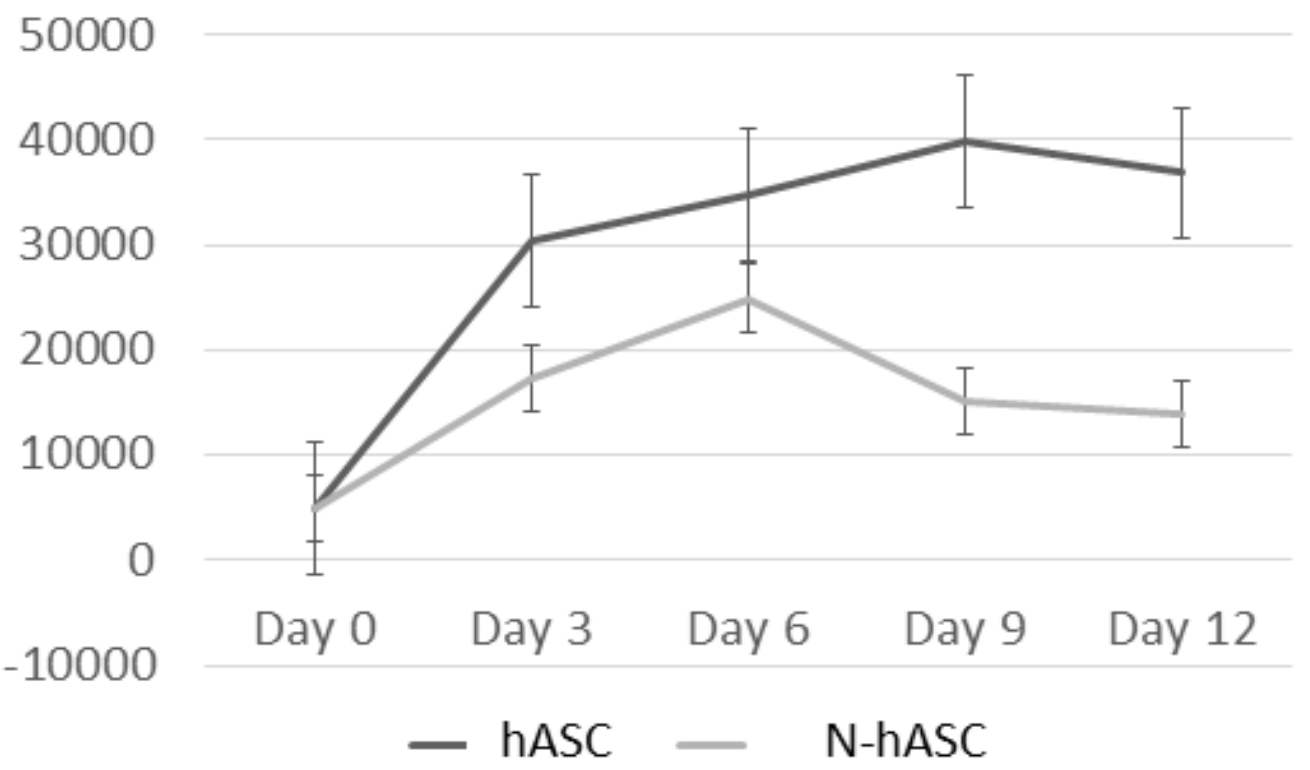

Figure 2 
Representative images and growth curves of hASC and NhASC. 2A. Representative images of hASC (left panel) and NhASC (right panel) exposed to either standard culture media or neurobasal differentiation media, respectively, after 15 day of development. NhASC present lower confluence at 15 day of development through the exposure of neurobasal media differentiation compared with the hASC at the same day of development at standart culture conditions. 2B. Standard growth curve conducted from 0 to 12 days suggests lower proliferative capacity of NhASC compared to hASC.

3A

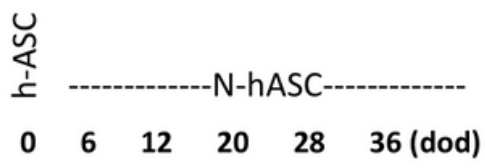

MAP-2

$150 \mathrm{kDa}$

bIII tubulin
$50 \mathrm{kDa}$

b-actin

$42 \mathrm{kDa}$
3B

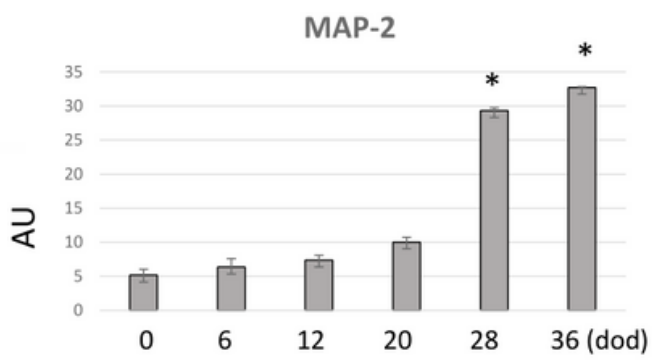

3C

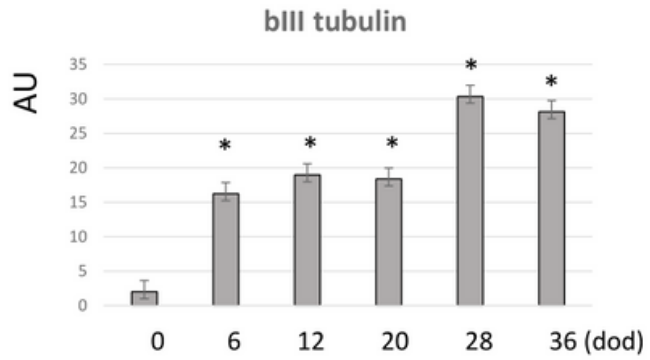

\section{Figure 3}

Representative blots and quantification of neuronal markers. 3A. Representative blots of MAP-2 and bIII tubulin neuronal markers protein expression in hASC and NhASC upon neuronal induction with neurobasal differentiation media over time, from 0 to 36 day of differentiation. From top to bottom, blots show signal of specific bands for neuronal MAP-2 and blll tubulin, and housekeeping b-actin (indicating similar protein loading in all samples). 3B. Quantification of MAP-2 neuronal marker protein expression upon neuronal induction ( $p<0.05$ in undifferentiated hASC at 0 day vs. 28 and 36 days of differentiation). 3C. Quantification of blll tubulin neuronal marker protein expression upon neuronal induction ( $<<0.05$ in hASC at 0 day vs. 6,28 and 36 days of development). AU, arbitrary units; Dod, day of differentiation; hASC, human adipose stem cells; NhASC, neuronal transdifferentiated human adipose stem cells. 
$4 \mathrm{~A}$

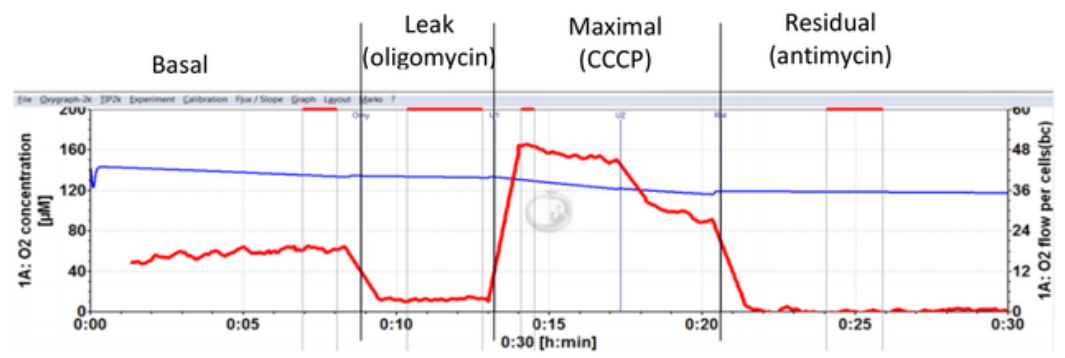

4B

4C
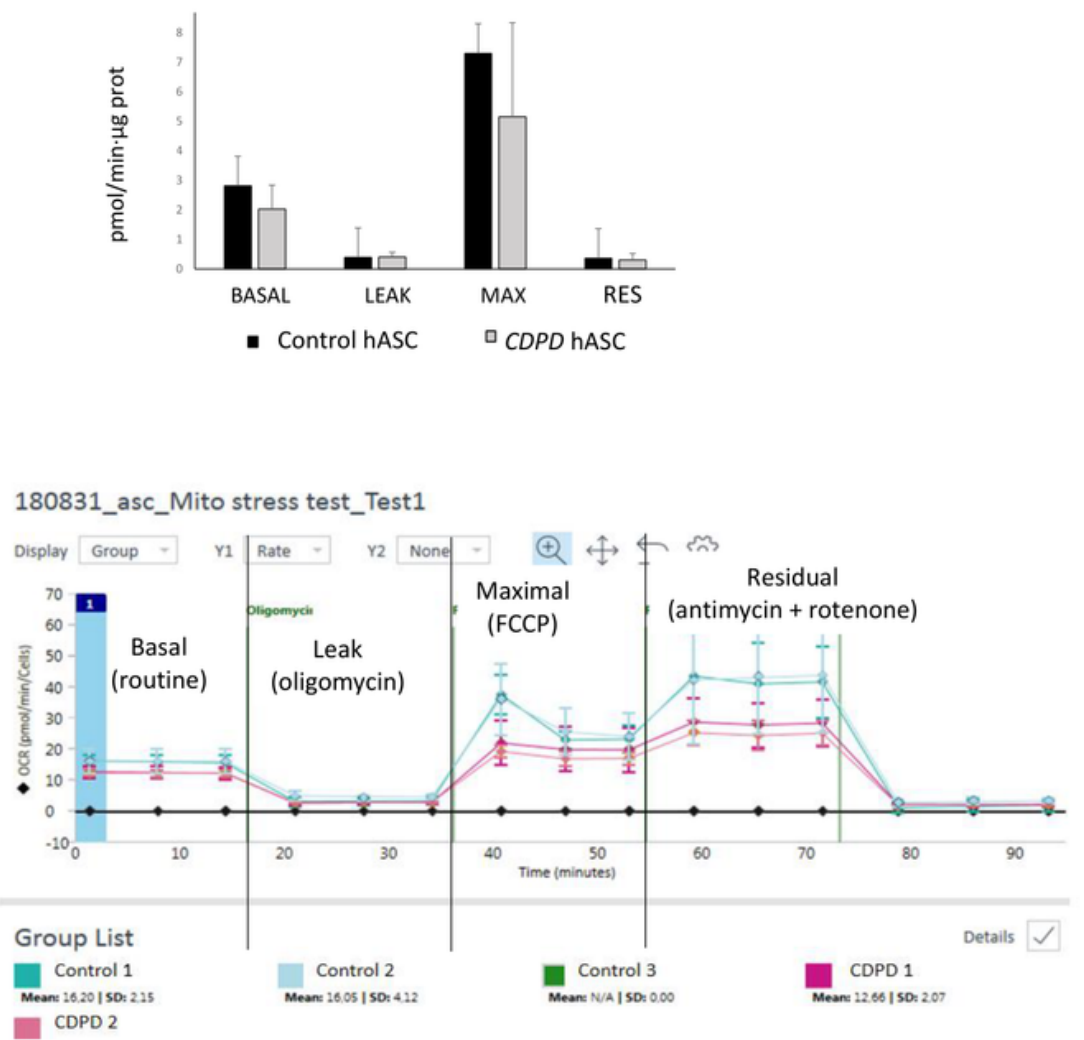

4D

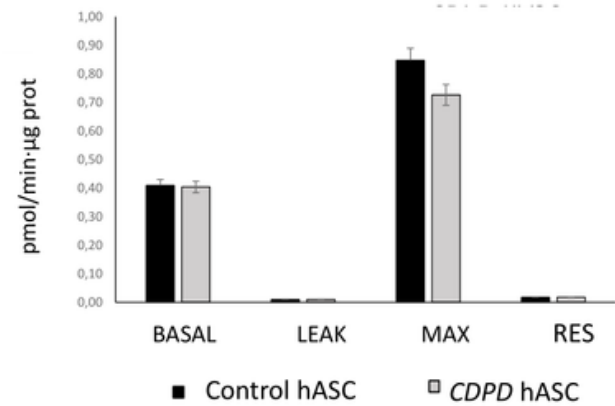

\section{Figure 4}

Bioenergetics profile through high resolution respirometry. 4A. Representative respiration OXPHOS slope states by Oroboros $2 \mathrm{k}$-Oxygraph. Substrate and coupling states are shown. Blue curve indicates 02 concentration in 02k chamber, oxygen fluxes per chamber volume are depicted (red curve) for 02k chamber operated simultaneously. The upper horizontal bar denotes the respiratory states (Routine, OXPHOS, Leak and electron transport system state. 4B. Respirometry assessment and quantification of healthy control hASC vs. CDPD hASC. Basal or routine respiration of intact cells without any substrates or inhibitors, proton 
leak after oligomycin inhibitor of ATP synthase or complex $\mathrm{V}$, maximal respiratory capacity after CCCP uncoupler addition and final residual respiration after antimycin inhibition of the mitochondrial respiratory chain (specific inhibition of complex III). Basal oxidative respiration of intact cells trends to lower respiration levels in the CDPD cases $(p=0.1)$. 4C. Representative respiration slope states by Seahorse X24 Agilent technology. Substrate and coupling states are shown in triplicates simultaneously, through the 24-well system. 4D. Respirometry assessment and quantification of healthy control hASC vs. CDPD hASC. The same profile of basal respiration, proton leak after oligomycin inhibition, maximal respiratory capacity after FCCP uncoupler titration and final residual respiration after rotenone-antimycin inhibition of the mitochondrial respiratory chain (specific inhibition of complex I and III, respectively) is shown. Basal Respiration was calculated as (Basal-Rote/AA), Maximal Respiration was calculated as (FCCP-Rote/AA). For both Oroboros and Seahorse technologies, trends towards lower maximal respiration capacity were observed in PD hASC. hASC, human adipose stem cells, CDPD, clinical diagnosed Parkinson's disease; Max, maximal; Res, residual.

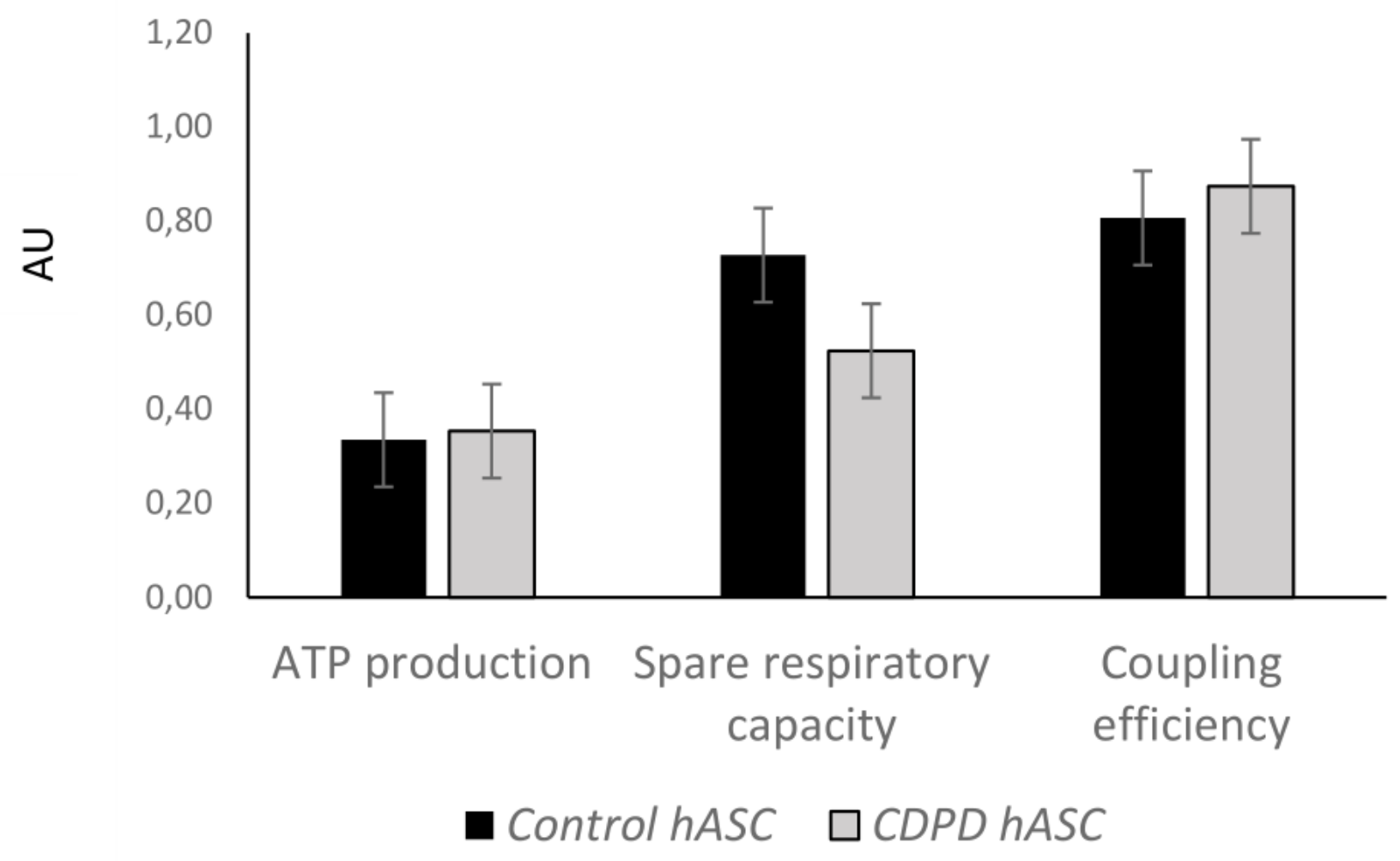

\section{Figure 5}

Oxidative parameters including ATP production, spare respiratory capacity and coupling efficiency of the mitochondrial respiratory chain. ATP production was calculated as (basal-H+ leak), spare respiratory capacity \% was calculated as (maximal/Basal) and coupling efficiency was calculated as (ATPprod/basal). Not significant differences were found between control and CDPD hASC lines. AU, arbitrary units; hASC, human adipose stem cells; CDPD, clinical diagnosed Parkinson's disease. 


\section{Supplementary Files}

This is a list of supplementary files associated with this preprint. Click to download.

- Additionalfile1.pdf 